\title{
Neuropathic Pain Causes Memory Deficits and Dendrite Tree Morphology Changes in Mouse Hippocampus
}

\section{Anna Tyrtyshnaia Igor Manzhulo (D)}

A.V. Zhirmunsky National Scientific Center of Marine Biology, Far Eastern Branch, Russian Academy of Sciences, Vladivostok, Russia
This article was published in the following Dove Press journal: Journal of Pain Research

Introduction: Neuropathic pain manifests in a diverse combination of sensory symptoms and disorders of higher nervous activity, such as memory deficiency, anxiety, depression, anhedonia, etc. This suggests the participation of brain structures, including the hippocampus, in the pathogenesis of neuropathic pain. The elucidation of central sensitization mechanisms underlying neuropathic pain cognitive and affective symptoms may be useful in the development of new and effective treatments for these common disorders. The study aims to elucidate the effect of chronic neuropathic pain on cognitive function and underlying neuronal plasticity in the hippocampus.

Methods: Chronic constriction injury of mouse right hind limb sciatic nerve was used as a model of neuropathic pain. The presence of neuropathic pain was confirmed by the thermal and mechanical allodynia. The morphology of the CA1 pyramidal neurons and the dentate gyrus (DG) granule neurons were studied using Golgi-Cox staining. The hippocampal proteins concentration was determined by immunohistochemistry and ELISA.

Results: Behavioral testing revealed reduced locomotor activity as well as impaired working and long-term memory in mice with a ligated nerve. We revealed changes in the dendritic tree morphology in CA1 and the dentate gyrus hippocampal subregions. We found the atrophy of the CA1 pyramidal neurons and an increase in the dendritic tree complexity in DG. Moreover, changes in the density of dendritic spines were observed in these regions. In addition, we revealed increased expression of the Arc protein in DG granule neurons and decreased surface expression of AMPA receptors within the hippocampus. Decreased AMPA receptors expression underlies observed altered dendrite arborization and dendritic spines morphology.

Discussion: We found that pain information entering the hippocampus causes neuronal plasticity changes. The changes in neurite arborization, dendritic length and dendritic spines morphology as well as protein expression are observed within the hippocampal regions involved in the processing of pain information. Moreover, changes in the dendrite morphology in hippocampal subregions are different due to the anatomical and functional heterogeneity of the hippocampus. Apparently, the detected morphological and biochemical changes can underlie the observed hippocampus-dependent behavioral and cognitive impairment in animals with neuropathic pain.

Keywords: neuropathic pain, chronic constriction injury, neurite arborization, dendrites, dendritic spines, mice

\section{Introduction}

Neuropathic pain, unlike ordinary pain, results from pathological excitation of neurons in the peripheral or central nervous system. The mechanisms of neuropathic pain are complex and not fully understood. The perception of pain and the transmission of
Correspondence: Igor Manzhulo Palchevskogo Str, 17, Vladivostok 69004I, Russia

Tel +79502967287

Email i-manzhulo@bk.ru 
a pain impulse is performed by the nociceptive system, which includes nerve fibers and pathways located in the spinal cord, as well as supraspinal centers. Nociceptive system includes several brain structures: the reticular formation, the thalamus nucleus, as well as the structures of the limbic system, including the hypothalamus, the amygdala, and the hippocampus. ${ }^{16}$ Patients with neuropathic pain experience symptoms characteristic of the limbic system impairment: anxiety, anhedonia, impaired spatial, episodic or contextual memory. ${ }^{14,17,25,29,30}$ However, the detailed mechanisms underlying neuropathic pain influence on the limbic system remain poorly understood. Given the key role of the hippocampal formation in cognitive functions, learning and memory, studying morphological, functional and plastic changes can help in the further search for the effective therapeutic agents to eliminate neuropathic pain consequences. The studies using various neuropathic pain models demonstrate changes in the hippocampus including impaired LTP, ${ }^{24}$ biochemical changes ${ }^{31}$ and impaired hippocampus-dependent functions, including working, ${ }^{43}$ shortterm $^{34}$ and recognition memory. ${ }^{12}$

In this study, we focused on changes in neuronal plasticity, which are the anatomical substrate of the observed functional changes. There is some evidence showing dendritic retraction in the hippocampus on various chronic stress models. However, the model of neuropathic pain cannot be fully considered as a model of chronic stress, because despite the presence of common symptoms, brain endophenotypes can vary. ${ }^{1}$ In addition, most of the chronic stress studies focus on the CA3 hippocampal area. ${ }^{27}$ Given that the perforant path is the main way of pain information entering into the hippocampus, including the projections from the layer II entorhinal cortex (EC) into the DG and from the layer III EC into the CA1 area, ${ }^{26}$ in this study we focused on these regions.

\section{Materials and Methods}

\section{Animals}

Male C57Bl/6 mice (30 $\pm 3 \mathrm{~g}, 3$-month-old) were used. The animals were raised at the National Scientific Center of Marine Biology, Far Eastern Branch of the Russian Academy of Sciences, Vladivostok, Russia. The mice were housed 2-5 per cage with ad lib access to food and water. Animals were maintained at a constant temperature $\left(23 \pm 2^{\circ} \mathrm{C}\right)$ and humidity $(55 \pm 15 \%)$ on daily 12 -h light/dark cycle. All procedures were approved by the Animal Ethics Committee at the National Scientific Center of Marine Biology, Far Eastern Branch, Russian Academy of Sciences (No 2/2019), according to the international regulations of the European Directive 2010/63/EU and ethical guidelines for the study of experimental pain in conscious animals by the International Association of the Study of Pain.

\section{Surgery}

Neuropathic pain was induced using the model of sciatic nerve chronic constriction injury (CCI). ${ }^{6}$ Animals were anesthetized with sodium pentobarbital (50 mg/kg, Sigma, St. Louis, MO, USA). The anesthetic was administered intraperitoneally, and surgical procedures began only after the stage of deep anesthesia in the animal. After the animal has been anesthetized, the right sciatic nerve was exposed and the three ligatures were placed closer to trifurcation with $1 \mathrm{~mm}$ between the ligatures (silk, Ethicon, USA). The ligatures were slightly tightened until a slight twitching of the limb appeared. All experimental animals were divided into 2 groups: "Sham" - sham-operated animals (20 animals); "CCI" - animals with CCI (20 animals). All mice were divided into groups randomly. The mice were sacrificed on the $21^{\text {th }}$ day after the surgery.

\section{Behavioral Testing}

Behavioral tests were performed 3 weeks after the surgery before mice sacrifice. All tests were performed during the light cycle between 7:00 A.M. and 7:00 P.M. Before testing each animal, the apparatus was thoroughly cleaned with $10 \%$ ethanol to minimize olfactory signals. In order to adapt the animals and avoid the stress associated with the new environment, the animals were placed in the test apparatus for 10 mins for 3 days preceding the day of testing. On the day of testing, the mice were left in their home cages in the room used for the experiment for 2 $\mathrm{h}$ before the onset of the behavioral study. Thermal allodynia measurement was carried out weekly.

\section{Thermal Allodynia}

Thermal allodynia was measured using a cold and a hot plate tests (Cold/Hot Plate Analgesia Meter Columbus Instruments, USA). ${ }^{3}$ The tests were performed in a chamber with acrylic walls $30 \mathrm{~cm}$ thick on a metal plate $30 \times 30 \mathrm{~cm}$. The temperature of the cold plate was $+4^{\circ} \mathrm{C}$, of the hot plate $+48^{\circ} \mathrm{C}$. Testing time was $60 \mathrm{~s}$. The mice were placed on the plate and the moment of the damaged hind paw first rise was recorded.

\section{Mechanical Hyperalgesia}

Mechanical hyperalgesia was evaluated using the pawpressure test. Mice were held gently, and progressive 
pressure was applied to the dorsal surface of the injured hind paw using the rodent pincher analgesia meter (Bioseb, USA/Canada) until a flexor response of the toes was observed. A cutoff of $450 \mathrm{~g}$ was set to prevent tissue damage. ${ }^{9}$

\section{Spontaneous Locomotor Activity}

Locomotor activity was evaluated by placing a mouse into the center of a clear Plexiglas circular open-field arena $(60 \mathrm{~cm}$ in diameter, $40 \mathrm{~cm}$ height) and allowing to explore for $5 \mathrm{~min}$. Bright overhead lighting was approximately 500 lux inside the arena. The area of the chamber was divided into 37 squares. Mouse behavior was continuously recorded by a video camera placed over the apparatus. In the subsequent analysis, the number of crossed squares was determined.

\section{Y-Maze Testing}

The working memory of experimental animals was studied in a Y-maze. The Y-maze was a device made of acrylic glass with three identical arms $(30 \mathrm{~cm} \times 10 \mathrm{~cm} \times 20 \mathrm{~cm})$. The mouse was placed in the center of the maze and left for 5 mins. The sequence of entries into the arms was recorded to calculate the spontaneous alternation rate. The criterion for entering the arms was the position of the mouse when all 4 paws were inside the arm. To calculate the spontaneous alternation rate, the following formula was used:

$$
K_{s}=R / A
$$

Ks - the spontaneous alternation rate, $\mathrm{R}$ - the number of consecutive entries into the three non-repeating arms, A the total number of possible alternations.

\section{Passive Avoidance Test}

The effects of chronic neuropathic pain on long-term memory was evaluated using a passive avoidance test. ${ }^{21}$ The testing apparatus consisted of light and dark compartments separated by a sliding door. In the training session, the mice were placed in the light compartment and allowed to explore for $60 \mathrm{~s}$ before the sliding door was opened. When the animal entered the dark compartment, the door was closed and $2 \mathrm{~s}$ later, an inescapable footshock $(0.3 \mathrm{~mA}, 2 \mathrm{~s})$ was delivered. The test session was performed $24 \mathrm{~h}$ after the training session without the footshock. The step-through latency for animals to enter the dark compartment was measured.

\section{Golgi-Cox Staining}

Animals were anesthetized with an overdose of sodium pentobarbital (60 mg/kg, Sigma, St. Louis, MO, USA). The anesthetic was administered intraperitoneally, and surgical procedures began only after the stage of deep anesthesia in the animal. After the animal has been anesthetized, brains were removed quickly from the skull, rinsed with 0.1M PBS $\left(+4^{\circ} \mathrm{C}\right)$ and cut into 2 hemispheres. The material was stained with the FD Rapid GolgiStain ${ }^{\mathrm{TM}}$ kit (FD NeuroTechnologies, Ellicott City, MD, USA) according to manufacturer's instructions (http://www.fdneurotech.com/item/0/41/0/733/FD Rapid_GolgiStain_Kit_large). Cryomicrotome (HM 550, Thermo Scientific, USA) was used to cut $100 \mu$ m thick slices. Slices were mounted on a gelatin-coated microscope slides, stained, dehydrated and coverslipped with VectaMount ${ }^{\mathrm{TM}}$ Mounting Medium (H-5000, Vector, USA).

\section{Sholl Analysis}

We performed a Sholl analysis ${ }^{38}$ to evaluate the effect of neuropathic pain on the hippocampal dendrite morphology. ImageJ software (NIH, USA) was used for all image processing and morphological analyses. For dendrite tracing, images for each individual neuron were converted to 8-bit color images. Dendrites were traced using the NeuronJ plugin (http://www.imagescience.org/meijering/ software/neuronj/) as previously described. ${ }^{5}$ Sholl analysis was performed on the NeuronJ tracings using the Sholl Analysis plugin (http://fiji.sc/Sholl_Analysis). For the Sholl analysis, the single animals were selected as the unit of analysis (5 animals per group). For each animal, 2-3 well-stained neurons were taken for evaluation (separately for CA1 and DG). When calculating the density of dendritic spines, one neuron (15 per group) was used as the unit of analysis. For each mouse (5 mice per group) the evaluation was performed on 3 neurons. Both hemispheres were used to evaluate the neuronal morphology.

\section{Immunohistochemical Studies}

Brains were extracted for subsequent immunohistochemical studies on the 21th day after surgery. The mice were anesthetized with sodium pentobarbital injection $(60 \mathrm{mg} / \mathrm{kg}$, Sigma, St. Louis, MO, USA). The anesthetic was administered intraperitoneally, and surgical procedures began only after the stage of deep anesthesia in the animal. Mice were transcardially perfused with $5 \mathrm{~mL}$ PBS $\left(\sim 4^{\circ} \mathrm{C}\right), \mathrm{pH}$ 7.2. Then the brain was rapidly removed from the skull, divided into 2 hemispheres and placed in 4\% paraformaldehyde for $12 \mathrm{hrs}$. Both hemispheres were used for immunohistochemical study. Afterward, the material was washed with PBS (pH 7.2). The tissue samples after paraformaldehyde fixation were embedded in paraffin blocks and sectioned at a thickness of $10 \mu \mathrm{m}$, using 
Leica rotary microtome RM 2245. The immunohistochemical method used in the study consists of the following steps: (1) Blocking endogenous peroxidase activity: $0.3 \% \mathrm{H}_{2} \mathrm{O}_{2}$ solution for $5 \mathrm{~min}$; (2) Blocks non-specific binding of antibodies: 5\% BSA in PBS for $1 \mathrm{~h}$; (3) Primary antibodies $\left(4^{\circ} \mathrm{C}, 24 \mathrm{hrs}\right)$; (4) Secondary antibodies labeled with horseradish peroxidase: PI1000 (anti-rabbit), 1:100 (Vector Laboratories, USA); (5) ImmPACT ${ }^{\mathrm{TM}}$ DAB Peroxidase Substrate chromogen (SK4105, Vector Laboratories, USA); (6) Washing with $0.1 \mathrm{M}$ PBS ( $\mathrm{pH}$ 7.2), dehydration and mounting in VectaMount Permanent Mounting Medium (H-5000, Vector laboratories). We used primary polyclonal rabbit antibodies to Glutamate Receptor 1 (AMPA subtype) (ab31232, Abcam, USA, 1:200), Arc (ab224717, Abcam, USA, 1:100).

Images were obtained on a Zeiss AxioScope A1 microscope equipped with an AxioCam 503 camera and AxioVision (Zeiss, Germany) software. Images were processed and analyzed using ImageJ software (NIH, USA). Processing of each micrograph included the following steps: conversion to black and white version (8-bit image); subtracting the background (rolling ball radius $=50$ ); contrast enhancement (+30 units); binarization. To measure the area of marker staining, the necessary area was selected, and the percentage of the colored area was calculated. When calculating Arc-immunopositive cells $/ \mathrm{mm}^{3}$, the formula was used: $\mathrm{d}=\left(10^{6 *} \mathrm{n}\right) /(\mathrm{S} * 1)$, where $\mathrm{n}-$ the number of cells; $\mathrm{S}$ - the area of the subgranule zone $\left(\mu \mathrm{m}^{2}\right) ; 1$ - the thickness of the slice, $10^{6}$ - the conversion coefficient $\mu \mathrm{m}^{2}$ to $\mathrm{mm}^{2}$. Counting Arcpositive cells was carried out in the granule layer of the DG. For statistical processing, the values obtained for each individual slice were averaged.

\section{Elisa}

Enzyme-linked immunosorbent assay (ELISA) was used to quantify the content of AMPA1 (GluR1) and Arc protein in the hippocampus. The mice were anesthetized with sodium pentobarbital (60 mg/kg, i.p.) and hippocampus was quickly removed, frozen in liquid nitrogen and stored at a temperature of $-70^{\circ} \mathrm{C}$. ELISA kits were used for detection of AMPA1 receptors (MBS9500254, MyBioSource, Inc., USA) and IL10 (ABIN1568489, antibodies-online GmbH, Germany) in accordance with the manufacturer's recommendations. Both hemispheres were used for ELISA. The hippocampal tissue was homogenized using a solution (100 mM Tris, $\mathrm{pH} 7.4,150$ $\mathrm{mM} \mathrm{NaCl}, 1 \mathrm{mM}$ EGTA, $1 \mathrm{mM}$ EDTA, 1\% Triton X-100, $0.5 \%$ sodium deoxycholate) with a cocktail of protease inhibitors (cOmplete ${ }^{\mathrm{TM}}$, Sigma-Aldrich) at a concentration of $1 \mathrm{mg} / \mathrm{mL}$. Protein concentration was determined using a BCA kit (Pierce, Rockford, IL). The optical density was measured using an iMark plate spectrophotometer (Bio-Rad) at a wavelength of $450 \mathrm{~nm}$.

\section{Statistical Analysis}

All values are presented as means \pm SEM. Normality of the data was evaluated with Shapiro-Wilk test. We used twosided Student's t tests or Mann-Whitney rank-sum tests for two-group comparisons (for datasets with abnormal distribution). $\mathrm{P}<0.05$ was considered significant. All statistical tests were performed using the Microsoft Excel software (Microsoft, USA) and GraphPad prism 4 (GraphPad Software, USA). For behavioral, immunohistochemical studies and Sholl analysis, the single animals were selected as the analysis unit. For dendritic spines density calculating, one neuron (15 per group) was used as the unit of analysis. For each mouse ( 5 mice per group) the evaluation was performed on 3 neurons. For ELISA we used the number of analyzed samples (15 samples per group, 3 replicates from each mouse) as the analysis unit.

\section{Results}

\section{Behavioral Effects of Neuropathic Pain}

To confirm neuropathic pain mice were tested for changes in thermal and mechanical sensitivity of the injured limb.

\section{Thermal Allodynia}

To determine thermal allodynia, we recorded the time before the injured limb was removed from the cold $\left(+4^{\circ} \mathrm{C}\right)$ and hot $\left(+48^{\circ} \mathrm{C}\right)$ plates. Testing on a hot plate showed the presence of thermal hypersensitivity as early as the first week after surgery. At the same time, at week 3 we observed an increase in sensitivity (Figure 1A). Cold plate tests revealed a sharp increase in cold hypersensitivity from the second week after surgery. A similar level of sensitivity persisted for 3 weeks (Figure 1B). The sham-operated group did not show allodynia in both tests.

\section{Mechanical Hyperalgesia}

Testing mechanical hyperalgesia using rodent pincher showed a hyperalgesic response already 1 week after the surgery. At week 3, hyperalgesia was not so pronounced, however, the values differed from the level of the Sham group $(\mathrm{p}<0.001)$. The sham-operated animals did not exhibit mechanical hyperalgesia (Figure 1C).

\section{Spontaneous Locomotor Activity}

We found decreased spontaneous locomotor activity in mice with neuropathic pain syndrome $1(101.90 \pm 9.68$ in 
A

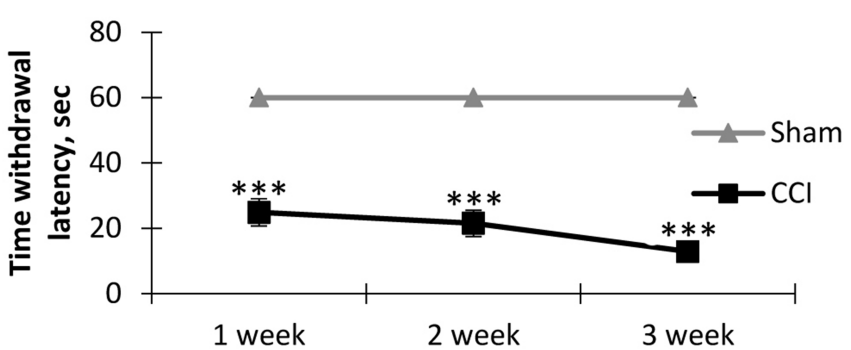

B

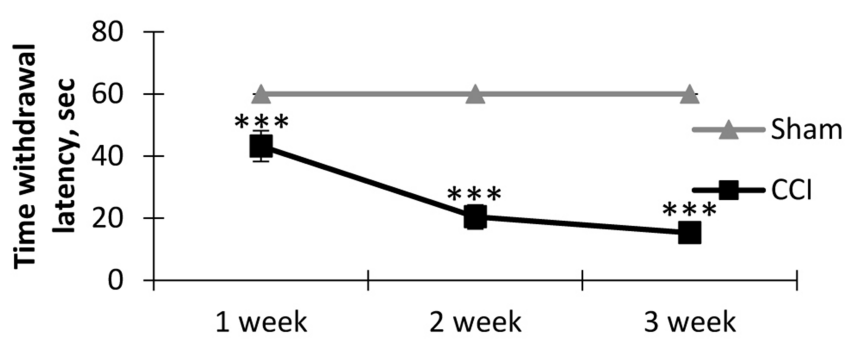

C

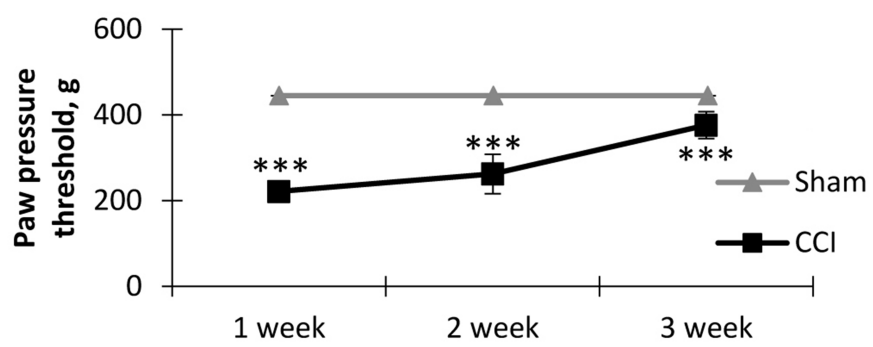

D

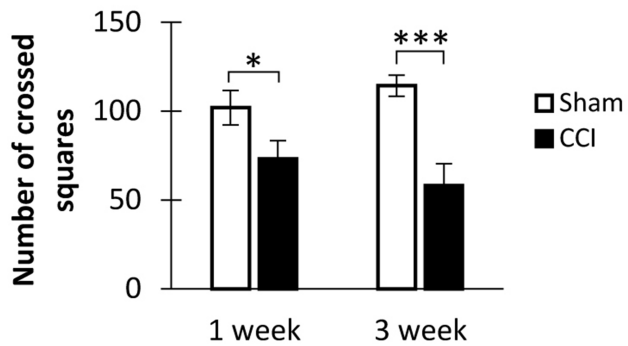

E

$\mathbf{F}$
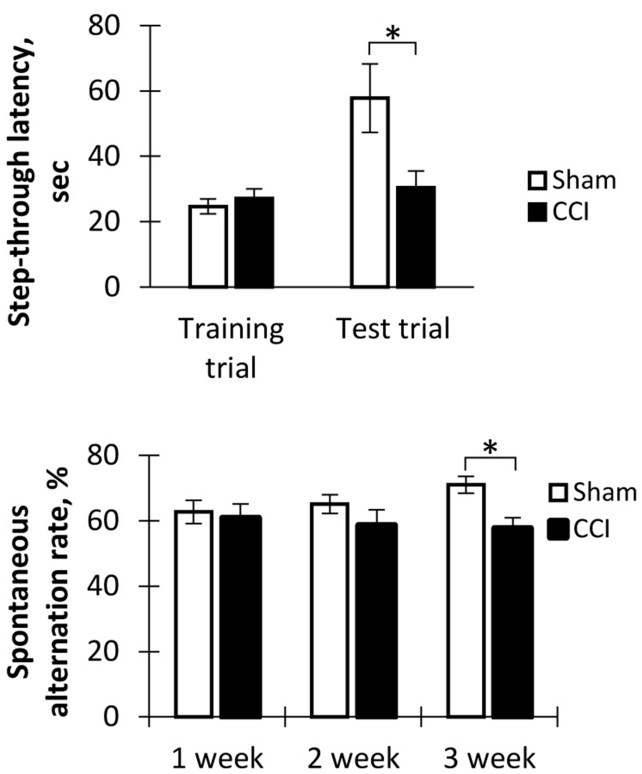

Figure I Behavioral effects of neuropathic pain. (A) The dynamics of hot allodynia: the moment of hind paw lifting above the hot plate $\left(+48^{\circ} \mathrm{C}\right)$ for the I-min observation, mean \pm SEM, $n=20$ (number of animals), $* * * p<0.00 I$. (B) The dynamics of cold allodynia: the moment of hind paw lifting above the cold plate $\left(+4^{\circ} \mathrm{C}\right)$ for the $1-\mathrm{min}^{\circ}$ observation, mean \pm SEM, $n=20$ (number of animals), ${ }^{* * *} p<0.001$. (C) The dynamics of mechanical hyperalgesia: the moment of flexor response of the toes after paw compression, mean $\pm S E M, n=20$ (number of animals), *** $p<0.001$. (D) Spontaneous locomotor activity in “open field", mean $\pm S E M, n=20$ (number of animals), *p=0.045, $*_{* *} p=0.0004$. (E) The step-through latency for animals to enter the dark compartment in passive avoidance test, mean $\pm S E M, n=20$ (number of animals), * $p=0.0147$. ( $F$ ) Spontaneous alternation rate in Y-maze, mean \pm SEM, $n=20$ (number of animals), ${ }^{*} \mathrm{p}=0.0147$.

Sham vs $73.20 \pm 10.21$ in CCI, $\mathrm{p}=0.045, \mathrm{n}=20)$ and 3 (114.30 \pm 5.93 in Sham vs 58.20 \pm 12.26 in CCI, $\mathrm{p}=0.0004$, $\mathrm{n}=20$ ) weeks after surgery (Figure 1D).

\section{Long-Term Memory}

A study of short-term memory in the passive avoidance test revealed no impairment in neuropathic pain. However, in the study of long-term memory ( $24 \mathrm{hrs}$ ), a decrease in the time interval before entering the dark chamber was observed in the CCI group compared to the Sham group $(57.80 \pm 10.45 \mathrm{sec}$ in Sham vs $31.00 \pm 5.37$ in CCI, $\mathrm{p}=0.0147, \mathrm{U}=18.00, \mathrm{n}=20$ ) (Figure 1E).

\section{Working Memory}

Working memory was evaluated using the test for spontaneous alternation in the Y-maze. We found a significant decrease in the spontaneous alternations rate in the CCI group at week 3 after the surgery $(71.00 \pm 2.55$-Sham vs $57.60 \pm 3.33$ - CCI - 3 weeks, $\mathrm{p}=0.0147, \mathrm{U}=15.50, \mathrm{n}=20$ ) (Figure $1 \mathrm{~F})$.

\section{Dendritic Morphology}

\section{CAI Pyramidal Neurons}

Sholl analysis showed a reduced complexity of CA1 pyramidal neurons in mice exposed to sciatic nerve ligation compared to sham-operated animals (Figure 2I). We found a decrease in the number of branches at $60-120 \mu \mathrm{m}$ from the soma in the CCI group compared to the Sham group $(\mathrm{p}<0.05)$ (Figure $2 \mathrm{~A})$. In addition, there was a decrease in the total number of branches $(42.60 \pm 5.35 \mu \mathrm{m}$ - Sham vs $23.60 \pm 4.33 \mu \mathrm{m}$ - CCI, $\mathrm{p}=0.0317, \mathrm{U}=2.00, \mathrm{p}<0.05, \mathrm{n}=5-$ number of animals) and the total length of the neurites $(1971.43 \pm 254.54 \mu \mathrm{m}$ - Sham vs $1198.51 \pm 166.49 \mu \mathrm{m}-\mathrm{CCI}, \mathrm{p}=0.0317, \mathrm{U}=2.00, \mathrm{p}<0.05, \mathrm{n}=5-$ 
A

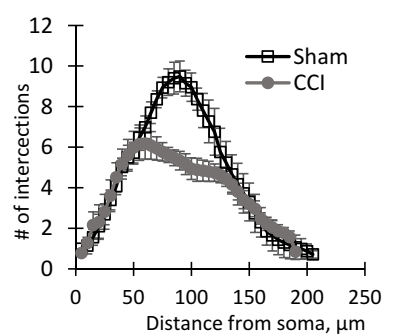

C

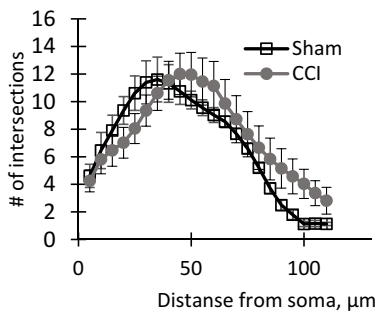

E

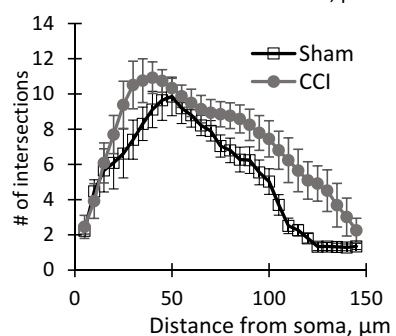

G

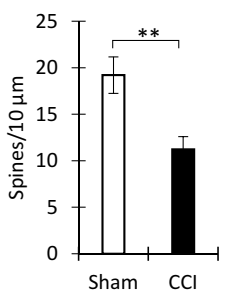

B

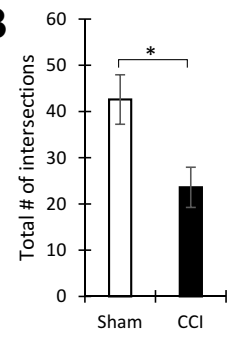

D

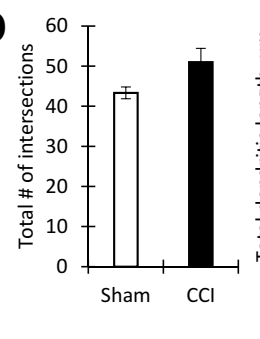

F

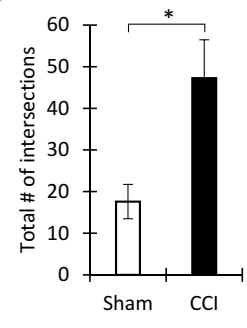

H
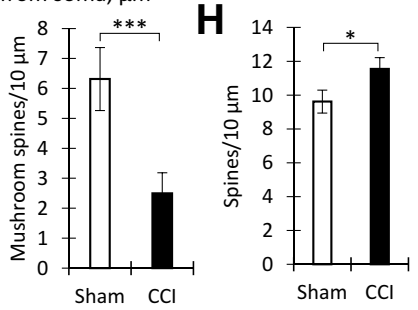
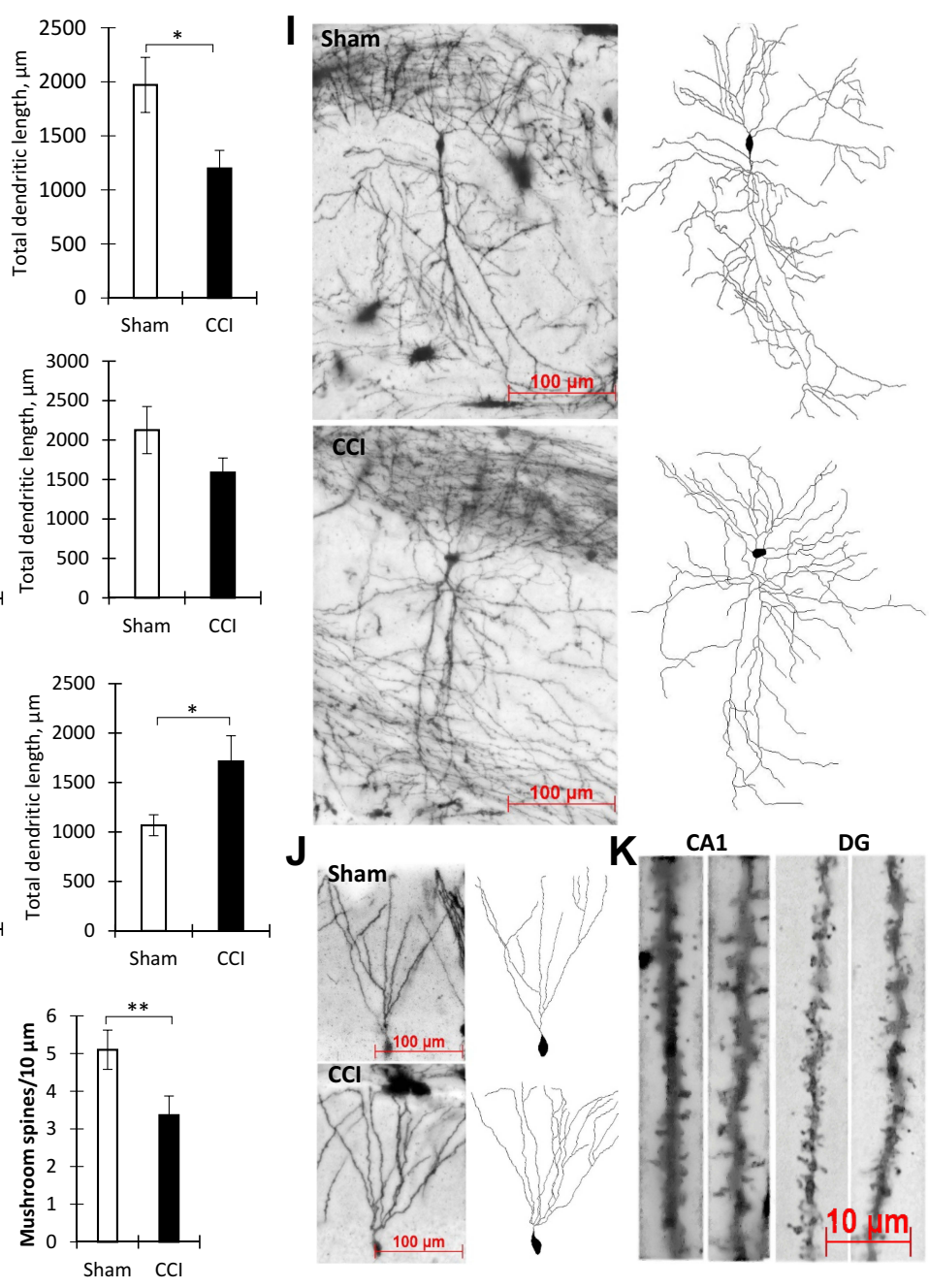

Figure 2 The results of dendrite Sholl analysis and spines density measurement. (A) Number of intersections along the apical dendritic trees at all distances from the soma in CAI pyramidal neurons, Mean \pm SEM, $n=5$ (number of animals). (B) The total number of branches (left, ${ }^{*} \mathrm{p}=0.03 \mathrm{I}$ ) and the total length of apical dendrites (right, ${ }^{*} \mathrm{p}=0.0317$ ) of CAI pyramidal neurons, $n=5$ (number of animals). (C) The number of intersections along the basal dendritic trees at all distances from the soma of $\mathrm{CAI}$ pyramidal neurons, $n=5$ (number of animals). (D) The total number of branches (left, $p=0.22$ ) and the total length of basal dendrites (right, $p=0.84$ ) of $C A I$ pyramidal neurons, $n=5$ (number of animals). (E) Number of Sholl intersections along the basal dendritic trees at all distances from the soma of $D G$ granule neurons, $n=5$ (number of animals). (F) The total number of branches (left, $\left.{ }^{*} \mathrm{p}=0.0159\right)$ and the total length of dendrites (right, $\left.{ }^{*} \mathrm{p}=0.0159\right)$ of $\mathrm{DG}$ granule neurons, $\mathrm{n}=5$ (number of animals). ( $\mathbf{G}$ ) Density of CAI pyramidal neurons dendritic spines: total - left (** $\left.*^{*}=0.0014\right)$, mushroom - right $(* * * P<0.0001)$, $n=15$ (number of analyzed neurons). (H) Density of DG granule neurons dendritic spines: total - left $\left({ }^{*} p=0.0283\right)$, mushroom - right $\left({ }^{* *} p=0.006\right), n=I 5$ (number of analyzed neurons). (I) The images of $C A I$ pyramidal neurons stained by the Golgi-Cox method from the Sham (upper) and CCl (lower) groups. (J) The images of DG granule neurons stained by the Golgi-Cox method from Sham (upper) and $\mathrm{CCl}$ (lower) groups. (K) The images of dendritic spines in the CAI-region and DG in Sham (left) and CCl (right) groups.

number of animals) (Figure 2B). In the CCI group, we observe increased branching in the basal dendrites of pyramidal CA1 neurons at $95-105 \mu \mathrm{m}$ from the soma $(\mathrm{p}<0.05)$ (Figure 2C). At the same time, the total number of branches and the total length of basal dendrites in the CCI group did not significantly change (Figure 2D).

Evaluation of the spines' density in the dendrites of CA1 pyramidal neurons showed a significant decrease in the CCI group (19.20 \pm 1.95 - Sham vs $11.21 \pm 1.34$ - CCI Spines/10 $\mu \mathrm{m}, \mathrm{p}=0.0014, \mathrm{U}=35.00, \mathrm{n}=15-$ number analyzed neurons). A decrease in the number of mushroom spines was also observed (6.31 \pm 0.10 - CCI vs $2.63 \pm 0.46$ - Sham, $\mathrm{p}<0.0001$,
$\mathrm{U}=18.00, \mathrm{n}=15-$ number of analyzed neurons) (Figure $2 \mathrm{G}$ and $\mathrm{K}$ ).

\section{DG Granule Neurons}

An increased number of granule DG neurons dendritic branches in the CCI group was observed at a distance of $105-140 \mu \mathrm{m}$ from the soma $(\mathrm{p}<0.05)$ (Figure $2 \mathrm{E}$ and $\mathrm{J}$ ). At the same time, the total number of branches in the CCI group was increased compared to Sham (17.60 \pm 4.13 - Sham vs $47.25 \pm 9.20$ - CCI, $\mathrm{p}=0.0159, \mathrm{U}=0.00, \mathrm{n}=5$ - number of animals). The total length of dendrites in DG granule neurons in the CCI group was also increased: 1107.96 \pm 105.52 - Sham 
vs $1713.46 \pm 259.24$ - CCI, $p=0.0159, \mathrm{U}=1.00$ ( $\mathrm{n}=5$ - number of animals) (Figure 2F). Evaluation of the spine's density on dendrites of granule DG neurons showed an increase in the total number of spines $(9.61 \pm 0.67$ - Sham vs $11.54 \pm 0.66$ CCI, $\mathrm{p}=0.0283, \mathrm{U}=64.00, \mathrm{n}=15$ - number analyzed neurons) and a decrease in the number of mushroom spines (5.12 \pm 0.50 - Sham vs $3.36 \pm 0.50$ - CCI, $\mathrm{p}=0.006, \mathrm{U}=41.50, \mathrm{n}=15$ (number analyzed neurons) (Figure $2 \mathrm{H}$ and $\mathrm{K}$ ).

\section{Hippocampal Protein Expression}

An immunohistochemical study of hippocampal protein expression revealed a decrease in the AMPARs expression in both the CA1 area $(10.56 \pm 1.02$ - Sham vs $6.02 \pm 1.24$ - CCI, $\mathrm{p}=0.0185, \mathrm{U}=19.00, \mathrm{n}=10$, number of animals) and the $\mathrm{DG}$ (3.71 \pm 0.32 - Sham vs $1.91 \pm 0.27$ - CCI, $p=0.0006, U=10.00$, $\mathrm{n}=10$, number of animals) (Figure $3 \mathrm{~A}$ and $\mathrm{C}$ ). In addition, an increase in the Arc protein production in the hippocampal DG was observed. A significant increase of Arc positive neurons was observed in the upper DG blade of the DG (770.94 \pm 103.75 - Sham vs 1406.21 \pm 216.44 - CCI, $p=0.0316$, $\mathrm{U}=120.00, \mathrm{n}=10$, number of animals). At the same time, no significant increase was observed in the lower bade (Figure 3B and D). The results of Arc protein quantification by IHC were confirmed by ELISA data, where a whole hippocampus was used for analysis (Figure 3F). However, ELISA reveled no significant decrease of AMPARs in the hippocampus (Figure 3E). As a result of endocytosis, a decrease in the density of receptors occurs only on the surface of the cell membrane. In this regard, we detected a decrease in the AMPARs density only by the IHC method.

\section{Discussion}

This study aims to evaluate neuronal plasticity in the hippocampi of mice with neuropathic pain. We found an increase in hippocampal Arc production. Arc protein accumulates rapidly with increasing neuronal activity, ${ }^{11}$ however, in our study, the presence of neuropathic pain led to a constantly increased level of this protein. The role of Arc in LTD and elimination of synapses is known, namely Arc translation regulates LTD induced by group 1 metabotropic glutamate receptors through AMPA-receptor endocytosis. ${ }^{4,45}$ A decrease in the density of AMPARs on the surface of DG granule neurons due to endocytosis, which, in turn, leads to a decrease in AMPA-mediated currents, $^{44}$ is probably associated with an increase in Arc

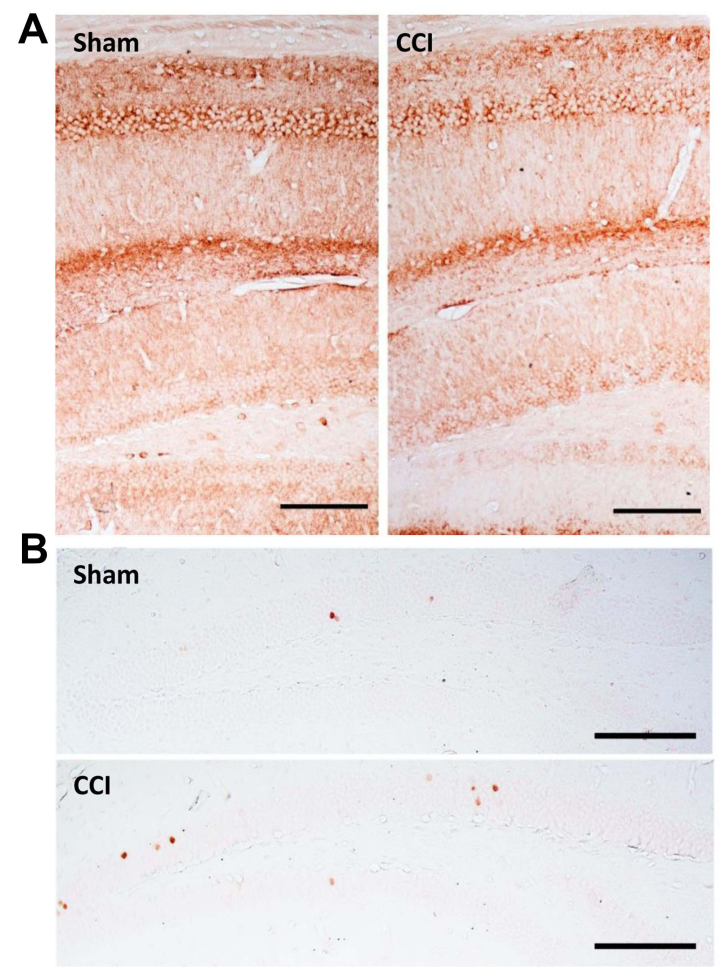

C
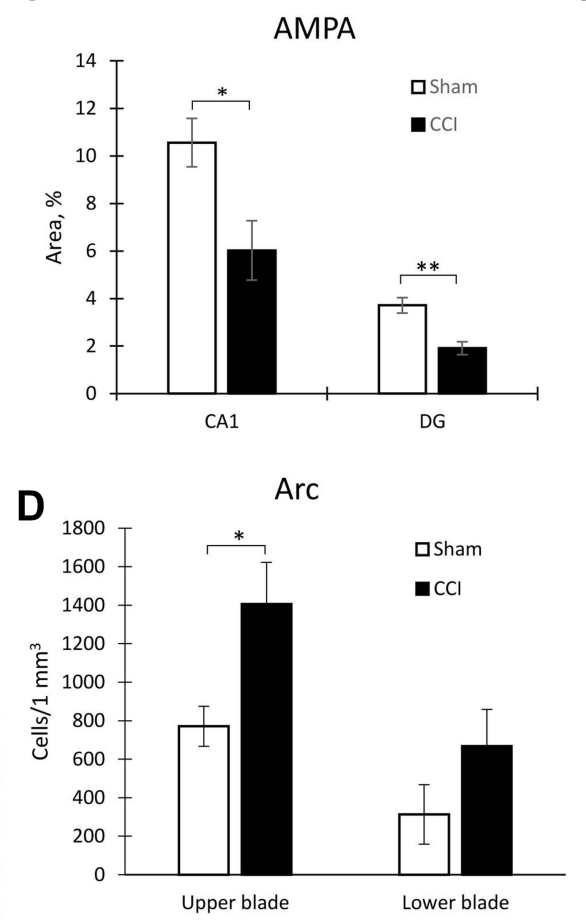

E

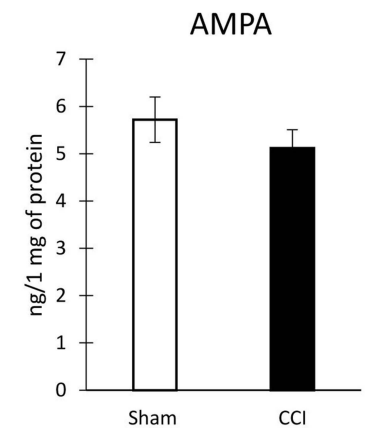

$\mathbf{F}$

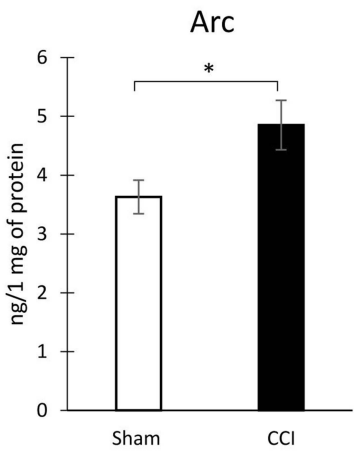

Figure 3 Hippocampal protein expression in Sham and CCl mice. Representative images of AMPARs (A) and Arc protein (B) immunohistochemical staining, Scale bar: 250 microns. (C) The percentage of area covered by AMPARs-positive staining within the hippocampal CAI (*p=0.0185) and DG areas (**p=0.0006) of Sham-operated and CCI mice, \%, $\mathrm{n}=10$ (number of animals). (D) The percentage of area covered by Arc-positive staining within the hippocampal DG (*p=0.03।6) of Sham-operated and CCI mice, \%, $n=10$ (number of animals). (E) The hippocampal AMPARs expression, $n=15$ (number of analyzed samples), $p=0.067$. (F) The hippocampal Arc expression, $n=15$ (number of analyzed samples), ${ }^{\mathrm{p}}=0.04$. 
production. AMPARs are of particular importance for altering the synaptic strength, as they mediate most of the fast excitatory synaptic transmission. ${ }^{10}$ Arc protein can regulate the number of postsynaptic glutamate receptors in postsynaptic density, the area of dendritic spines receiving presynaptic input. This submembrane protein network consists of cytoskeletal proteins, adhesion and signaling molecules. ${ }^{42}$ Thus, Arc protein, being regulated by neuronal activity and bound to the cytoskeleton proteins, link the synaptic activity and structuralfunctional plasticity of neurons. Similar changes underlie the detected behavioral effects. We found a peripheral neurotrauma-induced long-term memory loss. The disturbances of nonspatial processing in neuropathic pain indicates the involvement of the lateral EC neurons that terminate on the DG dendrites. Long-term memory is based on the modulation of synaptic connections and functional strengthening of existing synapses in response to significant impact. ${ }^{33}$ These processes are based on a change in dendritic spines morphology and trafficking of AMPARs. In addition to AMARs internalization, Arc induction changes the density of dendritic spines in DG granule neurons. We observe an increase in the total spines' density of DG granule cells dendrites with a simultaneous decrease in the density of mushroom spines, which can be achieved by increasing the density of thin spines. A similar picture was observed in a study by Peebles et al, ${ }^{32}$ where Arc increased spine density and regulated spine morphology by increasing the proportion of thin spines. It is worth noting that it is the mushroom spines that are responsible for the "storage of memories". This type of spine has a greater synaptic strength since it carries a greater number of AMARs and has a higher postsynaptic density. ${ }^{7}$ While thin spines constantly appear and disappear in response to a change in synaptic activity. ${ }^{19}$

In addition, we observe decrease in the number of branches and the total length of the CA1 pyramidal neurons apical dendrites. Dendritic atrophy is characteristic of many neurological pathologies associated with chronic stress. ${ }^{22,39}$ Such morphological changes are based on a decrease in AMPARs expression, impaired LTP $^{2}$ and a decrease in excitatory synaptic transmission to CA1 hippocampal neurons. ${ }^{37}$ A change in the dendritic tree morphology in the CA1 pyramidal neurons are associated with the observed disturbances of spatial orientation. Impaired working memory in $\mathrm{CCI}$ animals and the underlying morphological changes in the CA1 region dendritic tree results from the presence of direct axonal projections from layer III of the medial EC to the CA1 hippocampal area. The EC layer III inputs to the hippocampus is known to play a critical role in spatial working-memory tasks. ${ }^{23,41}$ This input as well as axons going from the EC layer II to the DG dendrites are the major input pathways of sensory information entering the hippocampus. ${ }^{26}$ The second input is critical for both spatial and nonspatial tasks, including memorizing objects, events, and contextual representation. ${ }^{13,28}$ The participation of these inputs in the pain signal transmission is evidenced by the observed long-term memory violations and other behavioral abnormalities demonstrated by several studies. For instance, a study by Mutso et al, ${ }^{29}$ demonstrates inability to extinguish context fear conditioning in animals with a spared nerve injury, and Cardoso-Cruz et al 2018 shows impaired working memory in rats after the sciatic nerve injury. In response of pain information input into the DG, the number of branches and the total dendritic length increases. We do not exclude the possibility that an increase in the length and complexity of dendrites in the DG granule neurons occurs as a compensatory reaction to a decrease in the number of newly formed $\mathrm{DCX}^{+}$neurons and a decrease in hippocampal neurogenesis, which we observed earlier in neuropathic pain animals. ${ }^{29,35,43}$ An increase in the dendritic tree complexity with a decrease in hippocampal neurogenesis explains the absence of short-term memory impairment. In addition, it is known that pathologies accompanied by chronic stress, ${ }^{20,40}$ including chronic neuropathic pain, are characterized by increased GABA release within the hippocampus. ${ }^{36} \mathrm{~A}$ local GABA increase in the hippocampal DG can induce the granule neuron arborization. ${ }^{15,18}$

\section{Conclusions}

Thus, we found that pain information entering the hippocampus causes neuroplastic changes. The abnormalities in neurite arborization, dendritic length and dendritic spines morphology are observed within the hippocampal regions involved in the processing of pain information. Moreover, changes in the dendrite morphology within the hippocampal subregions are different due to the anatomical and functional heterogeneity of the hippocampus. The detailed mechanisms of the revealed morphological changes are yet to be studied. However, it is clear that the key role is played by the Arc protein, participating in glutamatergic transmission and providing a link between synaptic activity and the structural-functional plasticity of neurons.

\section{Abbreviations}

CCI, chronic constriction injury; AMPA, $\alpha$-amino-3-hydroxy5-methyl-4-isoxazolepropionic acid; ELISA, enzyme-linked immunosorbent assay; DG, dentate gyrus; IHC, immunohisto- 
chemistry; PBS, phosphate buffer saline; DCX, doublecortin; PFA, paraformaldehyde.

\section{Data Sharing Statement}

The datasets used and analyzed during the current study are available from the corresponding author on reasonable request.

\section{Author Contributions}

AT conceptualized, wrote manuscript; performed the surgery, behavioral, histological and ELISA experiments, and image analysis. IM performed the surgery and behavioral and immunohistochemical studies. Both authors contributed to data analysis, drafting or revising the article, gave final approval of the version to be published, and agree to be accountable for all aspects of the work.

\section{Ethics Approval}

All procedures were approved by the Animal Ethics Committee at the National Scientific Center of Marine Biology, Far Eastern Branch, Russian Academy of Sciences (No 2/2019), according to the international regulations of the European Directive 2010/63/EU and ethical guidelines for the study of experimental pain in conscious animals by the International Association of the Study of Pain.

\section{Funding}

This study is supported by the Russian Science Foundation (project \# 17-74-20006).

\section{Disclosure}

Dr Igor Manzhulo reports grants from Russian Science Foundation, during the conduct of the study. The authors declare no other conflicts of interest.

\section{References}

1. Abdallah CG, Geha P. Chronic pain and chronic stress: two sides of the same coin? Chronic Stress. 2017;1:2470547017704763. doi: $10.1177 / 2470547017704763$

2. Alfarez DN, Joëls M, Krugers HJ. Chronic unpredictable stress impairs long-term potentiation in rat hippocampal CA1 area and dentate gyrus in vitro. Eur J Neurosci. 2003;17(9):1928-1934. doi:10.1046/j.1460-9568.2003.02622.x

3. Allen JW, Yaksh TL. Assessment of acute thermal nociception in laboratory animals. In: Luo DZ, editor. Pain Research: methods and protocols. Humana Press; 2004:11-23. doi:10.1385/1-59259-770$\mathrm{X}: 011$

4. Barylko B, Wilkerson JR, Cavalier SH, et al. Palmitoylation and membrane binding of Arc/Arg3. 1: a potential role in synaptic depression. Biochemistry. 2017;57(5):520-524. doi:10.1021/acs. biochem.7b00959
5. Bastian TW, Duck KA, Michalopoulos GC, et al. Eltrombopag, a thrombopoietin mimetic, crosses the blood-brain barrier and impairs iron-dependent hippocampal neuron dendrite development. J Thromb. 2017;15(3):565-574. doi:10.1111/jth.13602

6. Bennett GJ, Xie YK. A peripheral mononeuropathy in rat that produces disorders of pain sensation like those seen in man. Pain. 1988;33(1):87-107. doi:10.1016/0304-3959(88)90209-6

7. Bourne J, Harris KM. Do thin spines learn to be mushroom spines that remember? Curr Opin Neurobiol. 2007;17(3):381-387. doi:10.1016/j.conb.2007.04.009

8. Cardoso-Cruz H, Paiva P, Monteiro C, Galhardo V. Selective optogenetic inhibition of medial prefrontal glutamatergic neurons reverses working memory deficits induced by neuropathic pain. Pain. 2019;160(4):805-823. doi:10.1097/j.pain.0000000000001457

9. Célérier E, González JR, Maldonado R, Cabañero D, Puig MM. Opioid-induced hyperalgesia in a murine model of postoperative painrole of nitric oxide generated from the inducible nitric oxide synthase. Anesthesiology. 2006;104(3):546-555. doi:10.1097/ 00000542-200603000-00023

10. Chater TE, Goda Y. The role of AMPA receptors in postsynaptic mechanisms of synaptic plasticity. Front Cell Neurosci. 2014;8:401. doi:10.3389/fncel.2014.00401

11. Chowdhury S, Shepherd JD, Okuno H, et al. Arc/Arg3. 1 interacts with the endocytic machinery to regulate AMPA receptor trafficking. Neuron. 2006;52(3):445-459. doi:10.1016/j.neuron.2006.08.033

12. Kodama D, Ono H, Tanabe M. Increased hippocampal glycine uptake and cognitive dysfunction after peripheral nerve injury. Pain. 2011;152(4):809-817. doi:10.1016/j.pain.2010.12.029

13. Dees RL, Kesner RP. The role of the dorsal dentate gyrus in object and object-context recognition. Neurobiol Learn Mem. 2013;106:112-119. doi:10.1016/j.nlm.2013.07.013

14. Dick BD, Rashiq S. Disruption of attention and working memory traces in individuals with chronic pain. Anesth Analg. 2007;104 (5):1223-1229. doi:10.1213/01.ane.0000263280.49786.f5

15. Dioli C, Patrício P, Sousa N, et al. Chronic stress triggers divergent dendritic alterations in immature neurons of the adult hippocampus, depending on their ultimate terminal fields. Transl Psychiatry. 2019;9 (1):143. doi:10.1038/s41398-019-0477-7

16. Finnerup NB, Søren HS, Troels SJ. Chronic neuropathic pain: mechanisms, drug targets and measurement. Fundam Clin Pharmacol. 2007;21:129-136. doi:10.1111/j.1472-8206.2007.00474.x

17. Fishbain DA, Cutler R, Rosomoff HL, Rosomoff RS. Chronic pain-associated depression: antecedent or consequence of chronic pain? A review. Clin J Pain. 1997;13:116-137. doi:10.1097/ 00002508-199706000-00006

18. Ge S, Goh EL, Sailor KA, Kitabatake Y, Ming GL, Song H. GABA regulates synaptic integration of newly generated neurons in the adult brain. Nature. 2006;439(7076):589-593. doi:10.1038/nature04404

19. Holtmaat A, Wilbrecht L, Knott GW, Welker E, Svoboda K. Experience-dependent and cell-type-specific spine growth in the neocortex. Nature. 2006;441(7096):979. doi:10.1038/nature04783

20. Hu Y, Liao HB, Dai-Hong G, et al. Antidepressant-like effects of 3, 6'-disinapoyl sucrose on hippocampal neuronal plasticity and neurotrophic signal pathway in chronically mild stressed rats. Neurochem Int. 2010;56(3):461-465. doi:10.1016/j.neuint.2009.12.004

21. Ishiyama $T$, Tokuda $K$, Ishibashi $T$, et al. Lurasidone (SM-13496), a novel atypical antipsychotic drug, reverses MK-801-induced impairment of learning and memory in the rat passive-avoidance test. Eur J Pharmacol. 2007;572(2-3):160-170. doi:10.1016/j.ejphar.2007.06.058

22. Kallarackal AJ, Kvarta MD, Cammarata E, et al. (2013) Chronic stress induces a selective decrease in AMPA receptor-mediated synaptic excitation at hippocampal temporoammonic-CA1 synapses J. Neurosci. 2007;33(40):15669-15674. doi:10.1523/JNEUROSCI.2588-13.2013

23. Kitamura T, Macdonald CJ, Tonegawa S. Entorhinal-hippocampal neuronal circuits bridge temporally discontiguous events. Learn Mem. 2015;22(9):438-443. doi:10.1101/lm.038687.115 
24. Kodama D, Ono H, Tanabe M. Altered hippocampal long-term potentiation after peripheral nerve injury in mice. Eur J Pharmacol. 2007;574(2-3):127-132. doi:10.1016/j.ejphar.2007.07.054

25. Ling B, Coudoré-Civiale MA, Balayssac D, Eschalier A, Coudoré F, Authier N. Behavioral and immunohistological assessment of painful neuropathy induced by a single oxaliplatin injection in the rat. Toxicol. 2007;234(3):176-184. doi:10.1016/j.tox.2007.02.013

26. Liu MG, Chen J. Roles of the hippocampal formation in pain information processing. Neurosci Bull. 2009;25(5):237. doi:10.1007/ s12264-009-0905-4

27. McLaughlin KJ, Wilson JO, Harman J, et al. Chronic 17 $\beta$-estradiol or cholesterol prevents stress-induced hippocampal CA3 dendritic retraction in ovariectomized female rats: possible correspondence between CA1 spine properties and spatial acquisition. Hippocampus. 2010;20(6):768-786. doi:10.1002/hipo.20678

28. Morris AM, Weeden CS, Churchwell JC, Kesner RP. The role of the dentate gyrus in the formation of contextual representations. Hippocampus. 2013;23(2):162-168. doi:10.1002/hipo.22078

29. Mutso AA, Radzicki D, Baliki MN, et al. Abnormalities in hippocampal functioning with persistent pain. J Neurosci. 2012;32 (17):5747-5756. doi:10.1523/JNEUROSCI.0587-12.2012

30. Narita M, Kaneko C, Miyoshi K, et al. Chronic pain induces anxiety with concomitant changes in opioidergic function in the amygdala. Neuropsychopharmacol. 2006;31:739-750. doi:10.1038/sj.npp.1300858

31. Obata K, Yamanaka H, Dai Y, et al. Differential activation of extracellular signal-regulated protein kinase in primary afferent neurons regulates brain-derived neurotrophic factor expression after peripheral inflammation and nerve injury. $J$ Neurosci. 2003;23 (10):4117-4126. doi:10.1523/JNEUROSCI.23-10-04117.2003

32. Peebles CL, Yoo J, Thwin MT, et al. Arc regulates spine morphology and maintains network stability in vivo. PNAS Proc Natl Acad Sci. 2010;107(42):18173-18178. doi:10.1073/pnas.1006546107

33. Radwanska K, Medvedev NI, Pereira GS, et al. Mechanism for long-term memory formation when synaptic strengthening is impaired. PNAS Proc Natl Acad Sci. 2011;108(45):18471-18475. doi:10.1073/pnas.1109680108

34. Ren WJ, Liu Y, Zhou LJ, et al. Peripheral nerve injury leads to working memory deficits and dysfunction of the hippocampus by upregulation of TNF- $\alpha$ in rodents. Neuropsychopharmacol. 2011;36 (5):979-992. doi:10.1038/npp.2010.236

35. Romero-Grimaldi C, Berrocoso E, Alba-Delgado C, et al. Stress increases the negative effects of chronic pain on hippocampal neurogenesis. Anesth Analg. 2015;121(4):1078-1088. doi:10.1213/ ANE.0000000000000838
36. Saffarpour S, Shaabani M, Naghdi N, Farahmandfar M, Janzadeh A, Nasirinezhad F. In vivo evaluation of the hippocampal glutamate, GABA and the BDNF levels associated with spatial memory performance in a rodent model of neuropathic pain. Physiol Behav. 2017;175:97-103. doi:10.1016/j.physbeh.2017.03.025

37. Schmidt MV, Scharf SH, Liebl C, et al. A novel chronic social stress paradigm in female mice. Horm Behav. 2010;57(4-5):415-420. doi:10.1016/j.yhbeh.2010.01.010

38. Sholl DA. Dendritic organization in the neurons of the visual and motor cortices of the cat. J Anat. 1953;87(Pt 4):387-406.

39. Sousa N, Lukoyanov NV, Madeira MD, Almeida OF, Paula-Barbosa MM. Reorganization of the morphology of hippocampal neurites and synapses after stress-induced damage correlates with behavioral improvement. Neuroscience. 2000;97(2):253-266. doi:10.1016/ s0306-4522(00)00050-6

40. Stone DJ, Walsh JP, Sebro R, Stevens R, Pantazopolous H, Benes FM. Effects of pre-and postnatal corticosterone exposure on the rat hippocampal GABA system. Hippocampus. 2001;11(5):492-507. doi:10.1002/ hipo. 1066

41. Suh J, Rivest AJ, Nakashiba T, Tominaga T, Tonegawa S. Entorhinal cortex layer III input to the hippocampus is crucial for temporal association memory. Science. 2011;334(6061):1415-1420. doi:10.1126/ science. 1210125

42. Kaizuka T, Takumi T. Postsynaptic density proteins and their involvement in neurodevelopmental disorders. J Biochem. 2018;163 (6):447-455. doi:10.1093/jb/mvy022

43. Tyrtyshnaia AA, Manzhulo IV, Sultanov RM, Ermolenko EV. Adult hippocampal neurogenesis in neuropathic pain and alkyl glycerol ethers treatment. Acta Histochem. 2017;119:812-821. doi:10.1016/j. acthis.2017.10.007

44. Verde EM, Lee-Osbourne J, Worley PF, Malinow R, Cline HT. Increased expression of the immediate-early gene arc/arg3. 1 reduces AMPA receptor-mediated synaptic transmission. Neuron. 2006;52 (3):461-474. doi:10.1016/j.neuron.2006.09.031

45. Waung MW, Pfeiffer BE, Nosyreva ED, Ronesi JA, Huber KM. Rapid translation of Arc/Arg3. 1 selectively mediates mGluR-dependent LTD through persistent increases in AMPAR endocytosis rate. Neuron. 2008;59(1):84-97. doi:10.1016/j.neuron.2008.05.014
Journal of Pain Research

\section{Publish your work in this journal}

The Journal of Pain Research is an international, peer reviewed, open access, online journal that welcomes laboratory and clinical findings in the fields of pain research and the prevention and management of pain. Original research, reviews, symposium reports, hypothesis formation and commentaries are all considered for publication. The manuscript management system is completely online and includes a very quick and fair peer-review system, which is all easy to use. Visit http:// www.dovepress.com/testimonials.php to read real quotes from published authors. 